\title{
Coherent and incoherent ultrafast magnetization dynamics in $3 d$ ferromagnets driven by extreme terahertz fields
}

\author{
Mostafa Shalaby, ${ }^{1,2}$ Andreas Donges, ${ }^{3}$ Karel Carva,${ }^{4}$ Rolf Allenspach, ${ }^{5}$ Peter M. Oppeneer ${ }^{6}$ \\ Ulrich Nowak, ${ }^{3}$ and Christoph P. Hauri ${ }^{7,8}$ \\ ${ }^{1}$ Paul Scherrer Institute, CH-5232 Villigen PSI, Switzerland \\ ${ }^{2}$ Key Laboratory of Terahertz, Optoelectronics, Beijing Advanced Innovation Center for Imaging Technology CNU, Beijing 100048, China \\ ${ }^{3}$ Fachbereich Physik, Universität Konstanz, Universitätsstraße 10, D-78457 Konstanz, Germany \\ ${ }^{4}$ Charles University, Faculty of Mathematics and Physics, DCMP, Ke Karlovu 5, CZ-12116 Prague 2, Czech Republic \\ ${ }^{5}$ IBM Research-Zurich, Säumerstrasse 4, CH-8803 Rüschlikon, Switzerland \\ ${ }^{6}$ Department of Physics and Astronomy, Uppsala University, P.O. Box 516, SE-75120 Uppsala, Sweden \\ ${ }^{7}$ Ecole Polytechnique Fédérale de Lausanne, CH-1015 Lausanne, Switzerland \\ ${ }^{8}$ Paul Scherrer Institute, SwissFEL, CH-5232 Villigen PSI, Switzerland
}

(Received 31 July 2017; revised manuscript received 25 January 2018; published 3 July 2018)

\begin{abstract}
Ultrafast spin dynamics in magnetic materials is generally associated with ultrafast heating of the electronic system by a near infrared femtosecond laser pulse, thus offering only an indirect and nonselective access to the spin order. Here we explore spin dynamics in ferromagnets by means of extremely intense THz pulses, as at these low frequencies the magnetic field provides a direct and selective route to coherently control the magnetization. We find that, at low fields, the observed off-resonantly excited spin precession is phase locked to the $\mathrm{THz}$ magnetic field. At extreme $\mathrm{THz}$ fields, the coherent spin dynamics become convoluted with an ultrafast incoherent magnetic quenching due to the absorbed energy. This demagnetization takes place upon a single shot exposure. The magnetic properties are found to be permanently modified above a THz pump fluence of $\approx 100 \mathrm{~mJ} / \mathrm{cm}^{2}$. We conclude that magnetization switching cannot be reached. Our atomistic spin-dynamics simulations excellently explain the measured magnetization response. We find that demagnetization driven by $\mathrm{THz}$ laser-field coupling to electron charges occurs, suggesting nonconducting materials for achieving coherent THz-magnetization reversal.
\end{abstract}

DOI: 10.1103/PhysRevB.98.014405

\section{INTRODUCTION}

The reversal of a magnetic bit typically occurs on the nanosecond timescale in modern magnetic storage technology. Current developments aim at attaining reversal of the magnetic bit on a much faster, picosecond timescale, by means of an ultrashort laser pulse. To this end, femtosecond lasers operating in the near infrared (nIR) range $(v=400 \mathrm{THz})$ have been used to initiate ultrafast magnetization dynamics $[1,2]$. In this optically-induced ultrafast spin dynamics, the laser deposits energy in the electron system, immediately increasing its electron temperature, which subsequently decays into lattice and spin degrees of freedom [1-4]. However, this leads mostly to a transition to a paramagnetic phase and the goal of magnetization reversal is rarely achieved. Thus far, only a few studies, in particular, performed on a special class of rare earth-transition metal ferrimagnetic materials, showed deterministic single-shot magnetic switching on tens of picoseconds time scales [5-7]. Single shot all-optical switching was recently reported also for a thin $\mathrm{Pt} /$ Co stack [8]. For other materials, all-optical switching has only been achieved as a cumulative process by multishot exposure [9-11].

Over the past years, $\mathrm{THz}$ radiation has been proposed as an alternative route to ultrafast magnetization switching. It was conceived that the magnetic field acting at $\mathrm{THz}$ frequencies could lead to significantly faster switching in ferromagnets through direct coupling to the magnetic precessional motion without first heating the electrons [12].

The THz magnetic field directly couples to the spin degrees of freedom by the Zeeman interaction, which enables a highly efficient torque acting on the magnetic system, and potentially causes ultrafast switching without losing spin coherence properties. Thus far, state of the art table-top THz sources $[13,14]$, permitting time-resolved spin dynamics investigations, have been mainly restricted to precessional spin motion in the perturbative regime, that is, at low field excitation [15-20]. Coherent reversal of the spin orientation through precession has to date not been achieved, as it would require excitation with much stronger THz magnetic fields, i.e., several Teslas [21], which have only become available recently [22]. However, the heating effect associated with $\mathrm{THz}$ pulses was only considered recently. For this high-field regime, it was recently shown that $\mathrm{THz}$ excitation of Ni leads only to ultrafast demagnetization, with no clear experimentally observed trace of the desired precessional dynamics [23]. This demagnetization was facilitated by the low Curie temperature of $\mathrm{Ni}$ in comparison with other ferromagnets such as $\mathrm{Co}$ and Fe.

In this paper, we report spin dynamics measurements using tightly focused, intense $\mathrm{THz}$ bullets [22] exploring their potential for precessional switching in thin films of the classical conducting ferromagnets fcc Co and bcc Fe. We study the joint appearance of thermal and nonthermal contributions to 


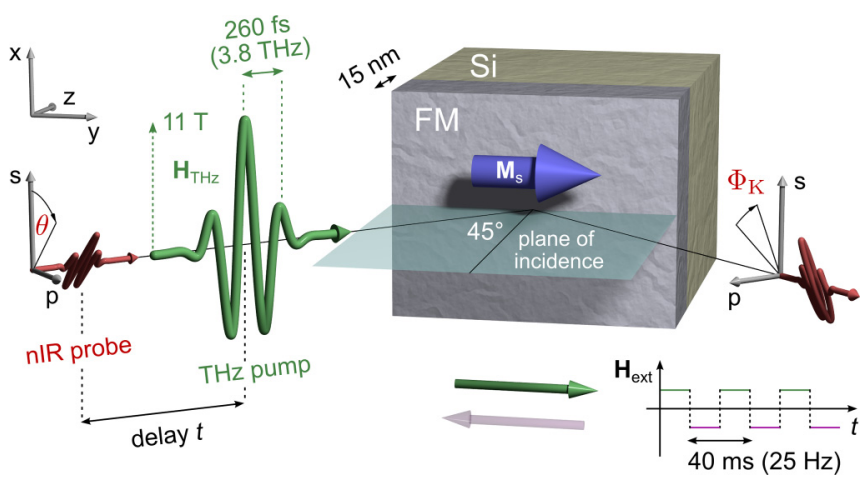

FIG. 1. Schematic diagram of the THz pump-magneto-optical probe setup used in the experiment. Both the linearly polarized $\mathrm{THz}$ pump and the delayed nIR optical probe ( $800 \mathrm{~nm}, 75 \mathrm{fs}$ ) collinearly impinge on the sample at an incidence angle of $45^{\circ}$. The sample is magnetized in-plane using an external $\mathrm{AC}$ magnetic field. The probe polarization angle $\theta$ has been set either to $0^{\circ}$ or $45^{\circ}$ with respect to s polarization, depending on the measurement. The reflected optical probe beam is sent to a balanced detector for Kerr rotation measurement. Experiments were carried out at room temperature.

the THz-induced magnetization dynamics. Using an advanced multiscale model, we show that coherent, precessional dynamics is indeed caused by the THz magnetic field while a heating process caused by the $\mathrm{THz}$ electric field absorption in the electronic system leads to incoherent demagnetization. Importantly, even for the strongest $\mathrm{THz}$ excitations, we do not achieve magnetization reversal, which suggests an immovable limit to the attainable coherent dynamics in $\mathrm{Fe}$ and $\mathrm{Co}$, and, hence, that for reaching coherent $\mathrm{THz}$ switching other material classes need to be considered.

\section{RESULTS}

To examine the THz-induced spin dynamics we performed two types of experiments. The first one is time-resolved THz pump-nIR magneto-optical Kerr effect (MOKE) probe to reveal the ultrafast dynamics. The second one is static spinpolarized scanning electron microscopy (spin-SEM) characterization [24] to study the spatial distribution of the permanent magnetic changes. We excite the $15 \mathrm{~nm}$ magnetic thin films with the ultra-intense $\mathrm{THz}$ bullet (see [22]). The laser system was operating with a repetition rate of $100 \mathrm{~Hz}$. The configuration of the THz pump and nIR probe beams at the sample position is shown schematically in Fig. 1. To orient the magnetization vector $\mathbf{M}$ of the film, an external magnetic field $\mu_{0} \mathbf{H}_{\mathrm{ext}}$ of $20 \mathrm{mT}$ was applied in the sample plane and parallel to the plane of incidence. To maximize the Zeeman torque and thus the precessional, coherent magnetization dynamics, we have chosen $\mathbf{M} \perp \mathbf{H}_{\mathrm{THz}}$. The external magnetic field was modulated at $25 \mathrm{~Hz}$ to eliminate any contributions from the nonmagnetic dynamics to the measured signal. Our acquisition system was locked to this frequency. The time-resolved magnetization dynamics was extracted by analyzing the MOKE signal in the reflected nIR probe beam. The probe polarization $\theta$ was set at an angle of $0^{\circ}$ or $45^{\circ}$ with respect to the s polarization.

To theoretically model the THz-radiation induced magnetization dynamics we employ a multiscale approach (see Sup-

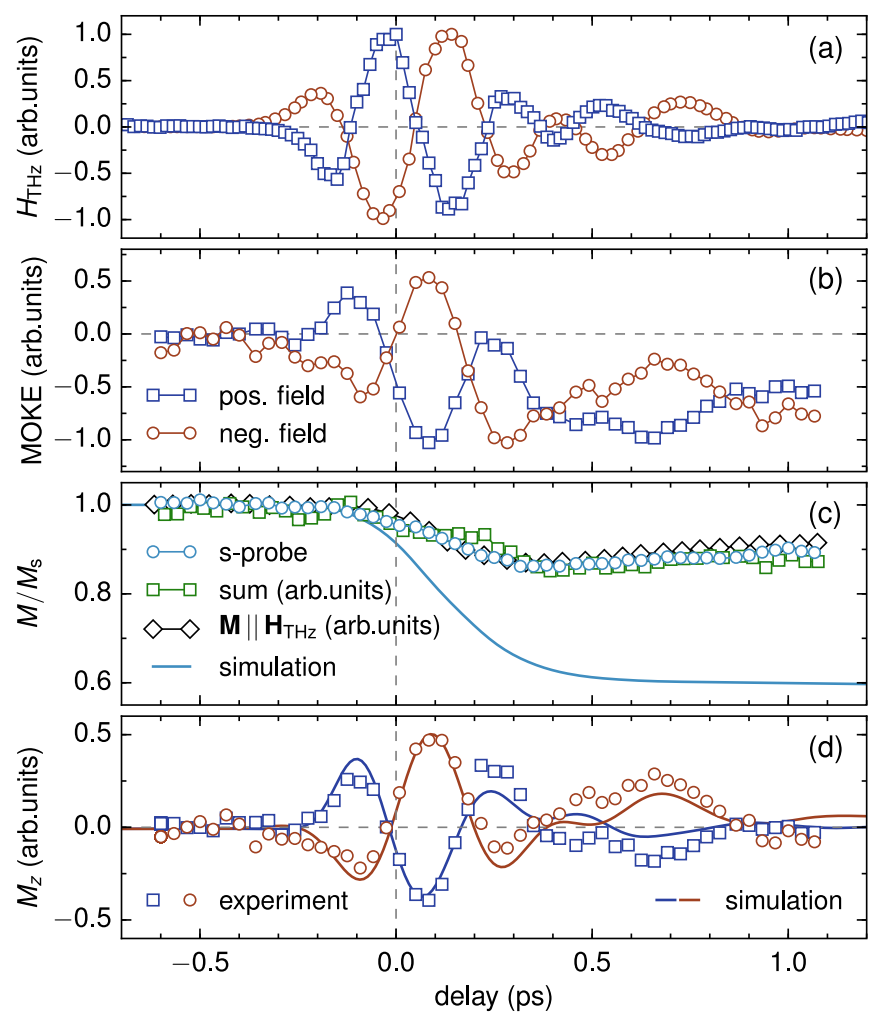

FIG. 2. Time-domain $\mathrm{THz}$ magnetic field and magnetization responses. (a) Total pulsed $\mathrm{THz}$ fields with opposite polarities. (b) THz-induced magnetization dynamics in Co under the excitation with the two pulses shown in (a) with peak magnetic field of 6.6 T. (c) Demagnetization components retrieved from different measurement techniques (see text) and atomistic simulations. (d) The extracted coherent precessional spin motion compared to calculated results from atomistic spin-dynamics simulations.

plemental Material (SM) [25] and Refs. [26,27]), in which we perform atomistic spin-dynamics simulations using input from $a b$ initio calculations to parametrize our spin Hamiltonian,

$$
\begin{aligned}
\mathcal{H}\left\{\mathbf{S}_{i}\right\}= & -\sum_{i j} J_{i j} \mathbf{S}_{i} \cdot \mathbf{S}_{j}-d_{z} \sum_{i} S_{i, z}^{2} \\
& -\mu_{0} \mu_{\mathrm{s}} \sum_{i} \mathbf{S}_{i} \cdot\left(\mathbf{H}_{\mathrm{ext}}+\mathbf{H}_{\mathrm{THz}}(t)\right) .
\end{aligned}
$$

Here, $J_{i j}$ denote the $a b$ initio calculated Heisenberg exchange constants, $d_{z}$ the uniaxial anisotropy, and $\mathbf{S}_{i}=\mu_{i} / \mu_{\mathrm{s}}$ the normalized magnetic moments. The Zeeman energy is given by the external $25 \mathrm{~Hz}$ AC-field $\mathbf{H}_{\text {ext }}$ and the $\mathrm{THz}$ magnetic field $\mathbf{H}_{\mathrm{THz}}(t)$ as depicted in Fig. 2(a) (for the conversion of the vacuum fields to the fields inside the Co film see the SM [25]). Since the amplitude of the quasiconstant field $H_{\text {ext }}$, which is used to saturate the magnetic film, is much smaller than the THz-magnetic field, the ultrafast spin dynamics is completely dominated by the latter. The spin dynamics in response to the $\mathrm{THz}$ pulse is computed by integrating the stochastic, atomistic Landau-Lifshitz-Gilbert (LLG) equation [27]. The effect of the THz electric field $\mathbf{E}_{\mathrm{THz}}$ is taken into account within the framework of an extended two-temperature model [1,28], via an excitation of the conduction electrons. The $\mathrm{THz}$ pulse excitation causes a rapid increase of the electronic temperature 

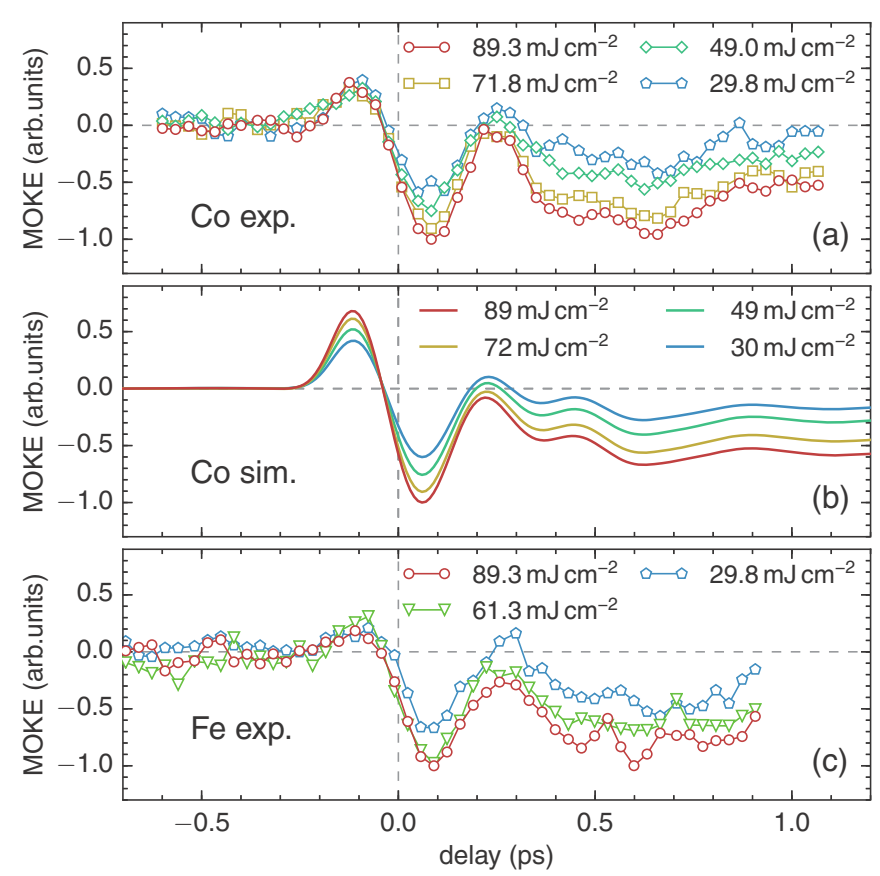

FIG. 3. Measurements (a) and simulations (b) of the total THzinduced magnetization dynamics in Co for different excitation fluences. Measurement on an Fe film (c), showing similar magnetization dynamics.

from which the heat is dissipated to the phonons and spin system (cf. [29]), causing an incoherent spin excitation and quenching of the magnetization. We use the transfer matrix method to calculate the absorbed $\mathrm{THz}$ energy to be $11 \%$ of the incident fluence. Finally, the calculated spin dynamics is then translated into the MOKE signal, as described in the SM [25]. The implementation of such a multiscale model is very time consuming and was performed only for fcc Co, since we do not expect significant differences for the Fe films, due to their similar magnetic properties.

The measured MOKE signal in the employed geometry with $\theta=45^{\circ}$ equally contains information on the in-plane and out-of-plane magnetization dynamics (see the SM [25] for details). The vectorial nature of coherent precessional dynamics suggests that the application of two stimuli with opposite signs [time-domain form shown in Fig. 2(a)] leads to opposite torques and thus reversed precessional dynamics. However, our measurement reveals a second distinct phenomenon, shown in Fig. 2(b). The arithmetic average of the total dynamics cancels out the coherent precession and thus represents the incoherent demagnetization contribution only, shown in Fig. 2(c). This conclusion is verified in two ways. First, changing the beam configuration such that $\mathbf{M} \| \mathbf{H}_{\mathrm{THz}}$, the torque component must vanish. Second, rotating the probe polarization angle $\theta$ from $45^{\circ}$ to $0^{\circ}$, the detected signal becomes most sensitive to the inplane magnetization, i.e., the demagnetization dynamics. All results excellently agree as shown in Fig. 2(c). The dependence of the dynamics on $\pm \mathbf{H}_{\mathrm{THz}}$ can also be used to isolate the pure precessional dynamics by subtracting the demagnetization from the total dynamics leading to the coherent spin motion shown in Fig. 2(d). The observed coherent dynamics are excellently reproduced by our multiscale simulations, whereas the demagnetization is overestimated in the simulations, see Fig. 2(c).

In Fig. 3 we show the fluence dependent measurements of both $\mathrm{Co}$ and $\mathrm{Fe}$, as well as the full numerical simulations for Co. We find that measurements and simulations equally show a continuous superposition of the fast coherent spin precession with the incoherent demagnetization; the latter becoming apparent by the shift of the MOKE signal towards negative values after the pulse has passed. The simulated out-of-plane precession due to the $\mathrm{THz}$ torque reaches a maximum of $10^{\circ}$ for a fluence of $89 \mathrm{~mJ} / \mathrm{cm}^{2}$, corresponding to $6.5 \mathrm{~T}$ in vacuum. The THz torque is strongest at the sample surface and becomes weaker with increasing depth. Thus, the average out-of-plane excursion over the whole film depth reaches only about $5^{\circ}$ at the center of the pump spot. From the measured magnetization dynamics in this geometry we can in itself not conclusively determine THz-induced magnetization reversal, however, our simulations show that the amount of precession is still far from switching. It should further be noted here that the precessional motion is not damped on this ultrashort time scale but simply stops after the $\mathrm{THz}$ pulse has passed and the effective field in the atomistic LLG equation is parallel to the magnetization again. At the same time, the demagnetization in the simulations reaches up to $40 \%$ at 1 ps pump-probe delay. This value is higher than the $14 \%$ that is observed in the experiment, as can be seen in Fig. 2(c). However, this is not unexpected since there are no adjustable parameters in our model and we do not take into account the field-induced changes of the absorption or additional dissipation channels, as, e.g., electron transport [30]. Furthermore, since in our two-temperature model simulations the Co film temperature at the center of the pump spot rises up almost to the Curie temperature, the amount of demagnetization is very sensitive to the absorbed fluence. Nevertheless, the simulated Kerr signal in Fig. 3(b), which is the weighted average over the whole probe spot, agrees well with the measurements shown in Fig. 3(a).

While the precessional motion is coherent, i.e., depending on the magnetic field polarity, the second effect, heat-induced demagnetization, is not as it depends primarily on the laser energy deposited in the electronic system. The heating of the spin system is indirect because it originates from the $\mathrm{THz}$ energy absorption by the electronic system, which is followed by a thermal energy exchange with the spin system. The latter results in a loss of coherence and demagnetization, the degree of which depends on the maximum electron temperature and the dynamics of the heating process. Both the coherent magnetic precession caused by the $\mathrm{THz}$ magnetic component and incoherent demagnetization caused by the $\mathrm{THz}$ energy absorption are launched within the temporal envelope of the THz pulse (Fig. 2). While the precessional spin motion is off resonant and locked to the $\mathrm{THz}$ magnetic field, the magnetization quenching persists over a much longer time scale.

For both materials, $\mathrm{Co}$ and $\mathrm{Fe}$, the effects of precession and demagnetization are evidently present for different fluences. Previous studies [16,31] suggested a linear dependence of the precessional excursion on the exciting $\mathrm{THz}$ magnetic field, whereas heat-induced demagnetization would suggest quadratic dependence on the electromagnetic stimulus. Therefore, as the $\mathrm{THz}$ intensity increases, the rate of heat-induced demagnetization quickly surpasses the precessional contribu- 


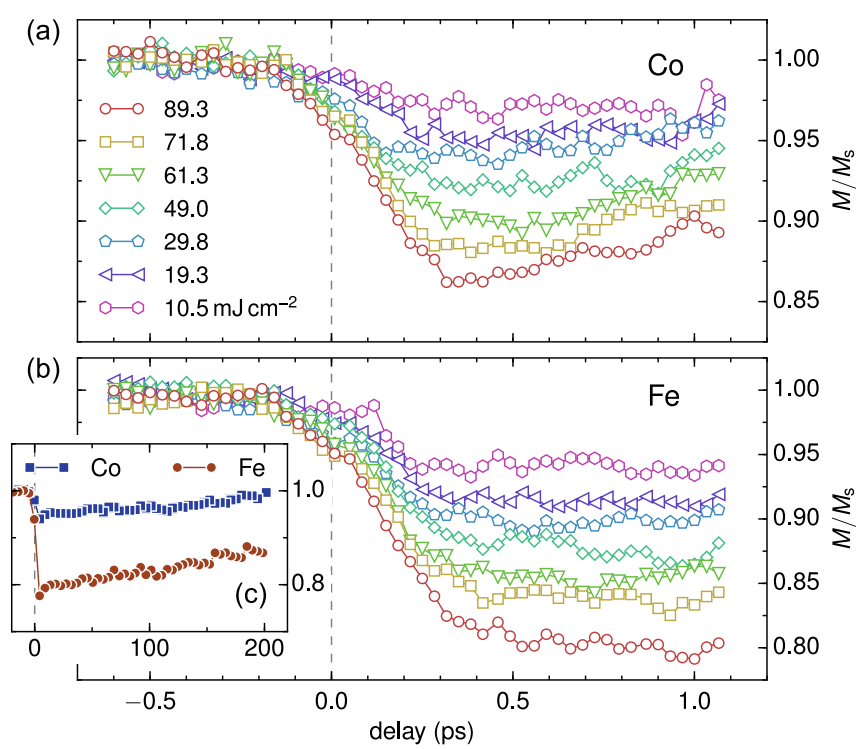

FIG. 4. Fluence-dependent THz-induced demagnetization in (a) cobalt and (b) iron. (c) The slow recovery of the demagnetization after excitation with a fluence of $89.3 \mathrm{~mJ} / \mathrm{cm}^{2}$.

tion to the measured dynamics. This effect is further enhanced by the critical behavior of the magnetization close to the Curie temperature.

To explore the THz-mediated demagnetization more thoroughly we modified the in-plane sensitivity of our MOKE setup by setting the probe polarization angle $\theta$ to $0^{\circ}$ where the demagnetization dynamics dominates the measured signal and the coherent spin dynamics is negligible. The ultrafast demagnetization measured with this $0^{\circ}$ setup, shown in Fig. 4, traces well the average of the $45^{\circ}$ setup shown in Fig. 2(c), where coherent and incoherent dynamics were simultaneously measured. Demagnetization occurs gradually as a function of the $\mathrm{THz}$ intensity as the deposited energy in the electronic system leads to an increase of the spin fluctuations towards criticality $\left(T_{\mathrm{C}}=1394 \mathrm{~K}\right.$ for $\mathrm{Co}, T_{\mathrm{C}}=1044 \mathrm{~K}$ for Fe [32]). The difference in $T_{\mathrm{C}}$ suggests that magnetization quenching is harder to induce in Co than in $\mathrm{Fe}$, neglecting the minor difference of the $\mathrm{THz}$ absorption coefficients. This qualitative picture is reflected in the quantitative measurements shown in Figs. 4(a) and 4(b). At a fluence of $89.3 \mathrm{~mJ} / \mathrm{cm}^{2}$, the amount of demagnetization reaches $14 \%$ for $\mathrm{Co}$ and $20 \%$ for $\mathrm{Fe}$, respectively. The ultrafast demagnetization coincides with the $\mathrm{THz}$ intensity envelope and occurs on the scale of $300 \mathrm{fs}$. The rate of demagnetization depends on the material and fluence and is slightly faster for cobalt. The recovery time extends beyond several hundreds of picoseconds [Fig. 4(c)].

The fluence dependence of the demagnetization is shown in Fig. 5(a). At low fluence, the measurements show nearly linear dependence. Here, the magnetization returns to its original state. Upon increasing the fluence beyond a critical value of ca. $100 \mathrm{~mJ} / \mathrm{cm}^{2}$ both $\mathrm{Fe}$ and Co begin to permanently lose their local magnetization. At a fluence of $252 \mathrm{~mJ} / \mathrm{cm}^{2}$ the demagnetization increases up to $23 \%$ and $28 \%$ for the Co and Fe films, respectively. For both samples the resulting saturation magnetization $\mathbf{M}_{\mathrm{s}}$ is reduced irreversibly, but the effect is less severe for Fe. The magnetization quenching is permanent and

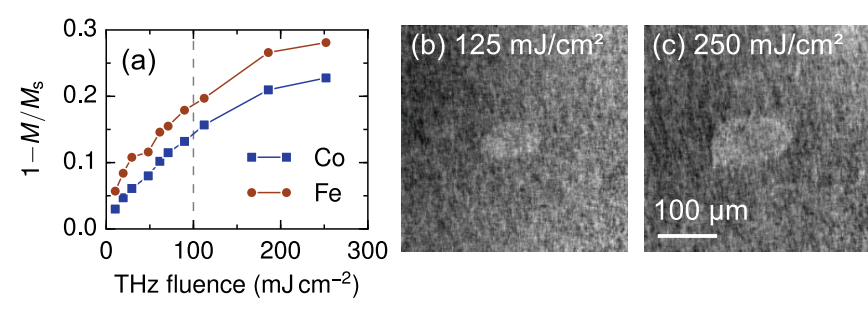

FIG. 5. Magnetization changes due to single-shot intense $\mathrm{THz}$ pulse excitation. (a) The measured fluence dependence of the peak demagnetization for $\mathrm{Co}$ and $\mathrm{Fe}$. The dashed line gives the threshold value beyond which permanent loss of magnetization happens. (b) and (c) Spin-SEM images of the Co film $M_{y}$ after a single shot $\mathrm{THz}$ exposure with different fluences.

continues to increase further with increase in the $\mathrm{THz}$ pump fluence. Furthermore, this effect is cumulative over time.

To determine the permanent changes in the Co sample we have employed spin-SEM, also known as SEMPA [24]. This technique is a variant of scanning electron microscopy to which a spin analyser is added. A focused beam of $8 \mathrm{keV}$ electrons scans along the surface, thereby exciting a wealth of low energetic secondary electrons $(0-20 \mathrm{eV})$ through electronelectron scattering. These electrons are ejected into vacuum and subsequently spin analyzed. The electron spin direction is a direct measure of the magnetization direction in the top $1 \mathrm{~nm}$ of the ferromagnet. In Figs. 5(b) and 5(c) we show two images taken at fluences of 125 and $250 \mathrm{~mJ} / \mathrm{cm}^{2}$ for a single $\mathrm{THz}$ shot after several weeks. In this measurement, the sample was first magnetized and then the external magnetic field was switched off before the $\mathrm{THz}$ bullet impinged on the sample. This measurement shows, first, that the demagnetization effect is permanent and can surprisingly be induced by a single $\mathrm{THz}$ shot. We suspect that the magnetization reduction occurs due to the formation of microdomains with opposite polarity (light-gray areas in Figs. 5(b) and 5(c), cf. [33]). Second, the change in magnetization is localized within the $\mathrm{THz}$ spot and sharp edges can be observed from the images.

\section{DISCUSSION AND CONCLUSIONS}

We start with noting that several THz-coupling channels could lead to the THz-induced demagnetization. First, THz phonon [34] excitation can be excluded due to the absence of phonons in our spectral excitation regime. Second, heating via direct magnon excitations (cf. [35]) can be excluded as well because we do not find a difference in the demagnetization dynamics upon changing the spin excitation symmetry from $\mathbf{M} \perp \mathbf{H}_{\mathrm{THz}}$ to $\mathbf{M} \| \mathbf{H}_{\mathrm{THz}}$ as shown in Fig. 2(c). Third, there is electron heating. Our spin-dynamics simulations confirm the importance of the THz-electric field electron heating. Here, for the classical ferromagnets $\mathrm{Co}$ and $\mathrm{Fe}$ we find that there is an upper limit to the $\mathrm{THz}$ fluence of ca. $100 \mathrm{~mJ} / \mathrm{cm}^{2}$ that can be maximally applied to these samples without causing irreversible demagnetization for the single cycle pulse used in our experiment [Fig. 2(a)]. Towards the target of THz switching, insulating magnetic materials look thus more promising than the conducting ferromagnetic ones studied here where the demagnetization effect can be significantly eliminated. Also, ferrimagnetic or antiferromagnetic materials $[15,21]$ could be 
more suitable for achieving $\mathrm{THz}$ switching. The switching might be facilitated using even lower THz-excitation frequency in compensated ferrimagnets [15]. We mention that a different, recently explored route to $\mathrm{THz}$-induced magnetization reversal could be to exploit local THz magnetic near-field enhancement in suitably fabricated nanostructures [36].

In conclusion, we have studied experimentally and numerically the fundamental interaction of low-frequency terahertz radiation with ferromagnetic $\mathrm{Co}$ and $\mathrm{Fe}$ up to extreme field strengths. We find that the spins coherently precess with the $\mathrm{THz}$ magnetic field while simultaneously demagnetization takes place at higher pump fluence. Using spin-SEM, we find that the demagnetization occurs upon a single $\mathrm{THz}$ shot and that permanent magnetization modification occurs above $100 \mathrm{~mJ} / \mathrm{cm}^{2}$ pump fluence. Our spin-dynamics simulations show that the demagnetization effect does not result from a direct heating channel due to coupling of the spins to the $\mathrm{THz}$ magnetic field. Rather, the heating of the spin system is mediated by the coupling of the conduction electrons to the $\mathrm{THz}$ electrical field. Our investigations thus establish an upper limit of the reachable THz-pumped spin excursion in classical ferromagnets and direct the search for even stronger, coherent magnetization dynamics to insulating ferri- or ferromagnets where the direct energy absorption of the electrons is minimal.

\section{ACKNOWLEDGMENTS}

M.S. acknowledges partial funding from the European Community's Seventh Framework Programme (FP7/2007-2013) under Grant Agreement No. 290605 (PSI-FELLOW/COFUND). A.D. and U.N. acknowledge support from the Center for Applied Photonics at Konstanz University. K.C. and P.M.O. acknowledge support from the Swedish Research Council (VR), the Röntgen-Ångström Cluster, the K. and A. Wallenberg Foundation (Grant No. 2015.0060) and the Swedish National Infrastructure for Computing (SNIC). C.P.H. acknowledges financial support from the Swiss National Science Foundation (SNSF) (Grants No. 200021_146769 and No. PP00P2_150732) and association to NCCR MUST. K.C. acknowledges support from the Czech Science Foundation (Grant No. 18-07172S). Lastly, we want to thank J. Hurst for performing COMSOL Maxwell-solver simulations.
[1] E. Beaurepaire, J.-C. Merle, A. Daunois, and J.-Y. Bigot, Phys. Rev. Lett. 76, 4250 (1996).

[2] A. Kirilyuk, A. V. Kimel, and T. Rasing, Rep. Prog. Phys. 76, 026501 (2013).

[3] J.-Y. Bigot, M. Vomir, and E. Beaurepaire, Nat. Phys. 5, 515 (2009).

[4] B. Koopmans, G. Malinowski, F. D. Longa, D. Steiauf, M. Fähnle, T. Roth, M. Cinchetti, and M. Aeschlimann, Nat. Mater. 9, 259 (2009).

[5] C. D. Stanciu, F. Hansteen, A. V. Kimel, A. Kirilyuk, A. Tsukamoto, A. Itoh, and T. Rasing, Phys. Rev. Lett. 99, 047601 (2007).

[6] I. Radu, K. Vahaplar, C. Stamm, T. Kachel, N. Pontius, H. A. Dürr, T. A. Ostler, J. Barker, R. F. L. Evans, R. W. Chantrell, A. Tsukamoto, A. Itoh, A. Kirilyuk, T. Rasing, and A. V. Kimel, Nature (London) 472, 205 (2011).

[7] T. A. Ostler, J. Barker, R. F. L. Evans, R. W. Chantrell, U. Atxitia, O. Chubykalo-Fesenko, S. E. Moussaoui, L. L. Guyader, E. Mengotti, L. J. Heyderman, F. Nolting, A. Tsukamoto, A. Itoh, D. Afanasiev, B. A. Ivanov, A. M. Kalashnikova, K. Vahaplar, J. Mentink, A. Kirilyuk, T. Rasing, and A. V. Kimel, Nat. Commun. 3, 666 (2012).

[8] M. Vomir, M. Albrecht, and J.-Y. Bigot, Appl. Phys. Lett. 111, 242404 (2017).

[9] S. Mangin, M. Gottwald, C.-H. Lambert, D. Steil, V. Uhliř, L. Pang, M. Hehn, S. Alebrand, M. Cinchetti, G. Malinowski, Y. Fainman, M. Aeschlimann, and E. E. Fullerton, Nat. Mater. 13, 286 (2014).

[10] C.-H. Lambert, S. Mangin, B. S. D. C. S. Varaprasad, Y. Takahashi, M. Hehn, M. Cinchetti, G. Malinowski, K. Hono, Y. Fainman, M. Aeschlimann et al., Science 345, 1337 (2014).

[11] R. John, M. Berritta, D. Hinzke, C. Müller, T. Santos, H. Ulrichs, P. Nieves, J. Walowski, R. Mondal, O. Chubykalo-Fesenko,
J. McCord, P. M. Oppeneer, U. Nowak, and M. Münzenberg, Sci. Rep. 7, 4114 (2017).

[12] I. Tudosa, C. Stamm, A. Kashuba, F. King, H. Siegmann, J. Stöhr, G. Ju, B. Lu, and D. Weller, Nature (London) 428, 831 (2004).

[13] M. Liu, H. Y. Hwang, H. Tao, A. C. Strikwerda, K. Fan, G. R. Keiser, A. J. Sternbach, K. G. West, S. Kittiwatanakul, J. Lu, S. A. Wolf, F. G. Omenetto, X. Zhang, K. A. Nelson, and R. D. Averitt, Nature (London) 487, 345 (2012).

[14] C. Riek, D. V. Seletskiy, A. S. Moskalenko, J. F. Schmidt, P. Krauspe, S. Eckart, S. Eggert, G. Burkard, and A. Leitenstorfer, Science 350, 420 (2015).

[15] T. Kampfrath, A. Sell, G. Klatt, A. Pashkin, S. Mährlein, T. Dekorsy, M. Wolf, M. Fiebig, A. Leitenstorfer, and R. Huber, Nat. Photon. 5, 31 (2010).

[16] C. Vicario, C. Ruchert, F. Ardana-Lamas, M. Derlet, B. Tudu, J. Lüning, and C. P. Hauri, Nat. Photon. 7, 720 (2013).

[17] M. Shalaby, C. Vicario, and C. P. Hauri, New J. Phys. 18, 013019 (2016).

[18] S. Bonetti, M. C. Hoffmann, M.-J. Sher, Z. Chen, S.-H. Yang, M. G. Samant, S. S. P. Parkin, and H. A. Dürr, Phys. Rev. Lett. 117, 087205 (2016).

[19] S.-W. Huang, E. Granados, W. R. Huang, K.-H. Hong, L. E. Zapata, and F. X. Kärtner, Opt. Lett. 38, 796 (2013).

[20] Z. Jin, A. Tkach, F. Casper, V. Spetter, H. Grimm, A. Thomas, T. Kampfrath, M. Bonn, M. Kläui, and D. Turchinovich, Nat. Phys. 11, 761 (2015).

[21] S. Wienholdt, D. Hinzke, and U. Nowak, Phys. Rev. Lett. 108, 247207 (2012).

[22] M. Shalaby and C. P. Hauri, Nat. Commun. 6, 5976 (2015).

[23] M. Shalaby, C. Vicario, and C. P. Hauri, Appl. Phys. Lett. 108, 182903 (2016).

[24] R. Allenspach, IBM J. Res. Dev. 44, 553 (2000). 
[25] See Supplemental Material at http://link.aps.org/supplemental/ 10.1103/PhysRevB.98.014405 for the theoretical modeling, including $a b$ initio calculations, atomistic spin model simulations of the coherent and incoherent magnetization dynamics and modeling of the magneto-optical response (see also Refs. [37-55]).

[26] D. Hinzke, U. Atxitia, K. Carva, P. Nieves, O. ChubykaloFesenko, P. M. Oppeneer, and U. Nowak, Phys. Rev. B 92, 054412 (2015).

[27] U. Nowak, Classical spin models, in Handbook of Magnetism and Advanced Magnetic Materials, edited by H. Kronmüller and S. Parkin (J. Wiley and Sons, New York, 2007).

[28] S. I. Anisimov, B. L. Kapeliovich, and T. L. Perelman, Zh. Eksp. Teor. Fiz. 66, 375 (1974).

[29] B. Frietsch, J. Bowlan, R. Carley, M. Teichmann, S. Wienholdt, D. Hinzke, U. Nowak, K. Carva, P. M. Oppeneer, and M. Weinelt, Nat. Commun. 6, 8262 (2015).

[30] We did not correct for the relatively long probe pulse duration in sampling the ultrafast transient. This leads to undersampling of the magnetization dynamics and thus a reduced sensitivity of the measurement MOKE signal. Furthermore, the centers of pump and probe spots might be misaligned by a few microns, reducing the effective fluence in the probe area.

[31] M. Shalaby, F. Vidal, M. Peccianti, R. Morandotti, F. Enderli, T. Feurer, and B. D. Patterson, Phys. Rev. B 88, 140301 (2013).

[32] E. P. Wohlfarth, Ferromagnetic Materials, edited by E. P. Wohlfarth, Handbook of Magnetic Materials, Vol. 1 (North-Holland, Amsterdam, 1980), pp. 1-70.

[33] C. Stamm, I. Tudosa, H. C. Siegmann, J. Stöhr, A. Y. Dobin, G. Woltersdorf, B. Heinrich, and A. Vaterlaus, Phys. Rev. Lett. 94, 197603 (2005).

[34] M. J. Verstraete, J. Phys.: Condens. Matter 25, 136001 (2013).

[35] S. V. Halilov, H. Eschrig, A. Y. Perlov, and P. M. Oppeneer, Phys. Rev. B 58, 293 (1998).
[36] D. Polley, M. Pancaldi, M. Hudl, P. Vavassori, S. Urazhdin, and S. Bonnetti, J. Phys. D: Appl. Phys. 51, 084001 (2018).

[37] I. Turek, V. Drchal, J. Kudrnovský, M. Šob, and P. Weinberger, Electronic structure of disordered alloys, surfaces and interfaces (Kluwer, Boston, 1997).

[38] U. von Barth and L. Hedin, J. Phys. C 5, 1629 (1972).

[39] A. I. Liechtenstein, M. I. Katsnelson, and V. A. Gubanov, J. Phys. F 14, L125 (1984).

[40] I. Turek, J. Kudrnovský, V. Drchal, and P. Bruno, Philos. Mag. 86, 1713 (2006).

[41] L. van Bockstal and F. Herlach, J. Phys.: Condens. Matter 2, 7187 (1990).

[42] J. Goddard and J. G. Wright, Br. J. Appl. Phys. 16, 1251 (1965).

[43] M. Born and E. Wolf, Principles of Optics, 6th ed. (Cambridge University Press, Cambridge, 1999).

[44] J. D. Jackson, Classical Electrodynamics, 3rd ed. (J. Wiley and Sons, New York, 1999).

[45] J. Dai, J. Zhang, W. Zhang, and D. Grischkowsky, J. Opt. Soc. Am. B 21, 1379 (2004).

[46] J. W. C. de Vries, Thin Solid Films 167, 25 (1988).

[47] Z. Lin, L. Zhigilei, and V. Celli, Phys. Rev. B 77, 075133 (2008).

[48] J. Wieczorek, A. Eschenlohr, B. Weidtmann, M. Rösner, N. Bergeard, A. Tarasevitch, T. O. Wehling, and U. Bovensiepen, Phys. Rev. B 92, 174410 (2015).

[49] R. Carey, B. W. J. Thomas, and D. M. Newman, Thin Solid Films 67, L35 (1980).

[50] H. H. Li, J. Phys. Chem. Ref. Data 9, 561 (1980).

[51] P. M. Oppeneer, in Handbook of Magnetic Materials, Vol. 13, edited by K. H. J. Buschow (Elsevier, Amsterdam, 2001), pp. 229-422.

[52] Z. J. Yang and M. R. Scheinfein, J. Appl. Phys. 74, 6810 (1993).

[53] F. Heiniger, E. Bucher, and J. Muller, Z. Phys. B 5, 243 (1966).

[54] T. Jarlborg and M. Peter, J. Magn. Magn. Mater. 42, 89 (1984).

[55] J. Zak, E. R. Moog, C. Liu, and S. D. Bader, Phys. Rev. B 43, 6423 (1991). 